УДК 517.5

\author{
A. O. Kuryliak, S. I. Panchuk, O. B. Skaskiv
}

\title{
BITLYAN-GOL'DBERG TYPE INEQUALITY FOR ENTIRE FUNCTIONS AND DIAGONAL MAXIMAL TERM
}

\begin{abstract}
A. O. Kuryliak, S. I. Panchuk, O. B. Skaskiv. Bitlyan-Gol'dberg type inequality for entire functi-
\end{abstract} ons and diagonal maximal term, Mat. Stud. 54 (2020), 135-145.

We obtain an analogue of Wiman-Bitlyan-Gol'dberg type inequality for entire $f: \mathbb{C}^{p} \rightarrow \mathbb{C}$ from the class $\mathcal{E}^{p}(\lambda)$ of functions represented by gap power series of the form

$$
f(z)=\sum_{k=0}^{+\infty} P_{k}(z), \quad z \in \mathbb{C}^{p} .
$$

Here $P_{0}(z) \equiv a_{0} \in \mathbb{C}, P_{k}(z)=\sum_{\|n\|=\lambda_{k}} a_{n} z^{n}$ is a homogeneous polynomial of degree $\lambda_{k} \in \mathbb{Z}_{+}$, and $0=\lambda_{0}<\lambda_{k} \uparrow+\infty(1 \leq k \uparrow+\infty), \lambda=\left(\lambda_{k}\right)$. We consider the exhaustion of the space $\mathbb{C}^{p}$ by the system $\left(\mathbf{G}_{r}\right)_{r \geq 0}$ of a bounded complete multiple-circular domains $\mathbf{G}_{r}$ with the center at the point $\mathbf{0}=(0, \ldots, 0) \in \mathbb{C}^{p}$. Define $M(r, f)=\max \left\{|f(z)|: z \in \bar{G}_{r}\right\}, \mu(r, f)=$ $\left.\max \left\{\mid P_{k}(z)\right) \mid: z \in \bar{G}_{r}\right\}$. Let $\mathcal{L}$ be the class of positive continuous functions $\psi: \mathbb{R}_{+} \rightarrow \mathbb{R}_{+}$such that $\int_{0}^{+\infty} \frac{d x}{\psi(x)}<+\infty, n(t)=\sum_{\lambda_{k} \leq t} 1$ the counting function of the sequence $\left(\lambda_{k}\right)$ for $t \geq 0$. The following statement is proved: If a sequence $\lambda=\left(\lambda_{k}\right)$ satisfy the condition

$$
\left(\exists p_{1} \in(0,+\infty)\right)\left(\exists t_{0}>0\right)\left(\forall t \geq t_{0}\right): \quad n(t+\sqrt{\psi(t)})-n(t-\sqrt{\psi(t)}) \leq t^{p_{1}}
$$

for some function $\psi \in \mathcal{L}$, then for every entire function $f \in \mathcal{E}^{p}(\lambda), p \geq 2$ and for any $\varepsilon>0$ there exist a constant $C=C(\varepsilon, f)>0$ and a set $E=E(\varepsilon, f) \subset[1,+\infty)$ of finite logarithmic measure such that the inequality

$$
M(r, f) \leq C m(r, f)(\ln m(r, f))^{p_{1}}(\ln \ln m(r, f))^{p_{1}+\varepsilon}
$$

holds for all $r \in[1,+\infty] \backslash E$.

The obtained inequality is sharp in general. In the case $\lambda_{k} \equiv k, p=2$ we have $p_{1}=1 / 2+\varepsilon$, therefore from obtained statement we get the assertion on the Bitlyan-Gol'dberg inequality (1959), and fot $p=1$ the classical Wiman inequality it follows.

1. Introduction. We use the following standard notation. Let $\mathbb{C}^{p}$ be the $p$-dimensional $(p \geq 1)$ a complex vector space, $\mathbb{Z}_{+}^{p}=(\mathbb{N} \cup\{0\})^{p}, z^{n}=z_{1}^{n_{1}} \cdots z_{p}^{n_{p}},\|n\|=n_{1}+\cdots+n_{p}$ for $n=\left(n_{1}, \ldots, n_{p}\right) \in \mathbb{Z}_{+}^{p}$ and $z=\left(z_{1}, \ldots, z_{p}\right) \in \mathbb{C}^{p}, \mathbb{R}_{+}=[0,+\infty)$. By $\mathcal{E}^{p}(\lambda)$ we denote of the class of entire functions $f: \mathbb{C}^{p} \rightarrow \mathbb{C}$, (i.e., entire functions of $p$ complex variables), represented by power series of the form

$$
f(z)=\sum_{k=0}^{+\infty} P_{k}(z), \quad z \in \mathbb{C}^{p}
$$

2010 Mathematics Subject Classification: 30B50.

Keywords: entire functions; several complex variables; dagonal maximal term; homogeneous polynomial. doi:10.30970/ms.54.2.135-145 
Here $P_{0}(z) \equiv a_{0} \in \mathbb{C}, P_{k}(z)=\sum_{\|n\|=\lambda_{k}} a_{n} z^{n}$ is a homogeneous polynomial of degree $\lambda_{k} \in \mathbb{Z}_{+}$, and $0=\lambda_{0}<\lambda_{k} \uparrow+\infty(1 \leq k \uparrow+\infty), \lambda=\left(\lambda_{k}\right)$. In the case $\lambda_{k} \equiv k(k \geq 0)$ we obtain the class of all entire functions of $p$ complex variables. Denote by $\mathcal{E}^{p} ; \mathcal{E}^{1}, \mathcal{E}^{1}(\lambda)$ the classes of entire functions of one variable and entire functions represented by gap power series of the form

$$
f(z)=a_{0}+\sum_{k=1}^{+\infty} a_{k} z^{\lambda_{k}}, \quad z \in \mathbb{C}
$$

respectively.

According to [1] we consider the exhaustion of the space $\mathbb{C}^{p}$ by a system $\left(\mathbf{G}_{r}\right)_{r \geq 0}$ of bounded complete multiple-circular domains with the center at the point $\mathbf{0}=(0, \ldots, 0) \in \mathbb{C}^{p}$. Actually, we assume that this system satisfies the conditions:

i) $\bigcup_{r \geq 0} \mathbf{G}_{r}=\mathbb{C}^{p}$

ii) $\left(\forall r_{1}<r_{2}\right): \mathbf{G}_{r_{1}} \subset \mathbf{G}_{r_{2}}$;

iii) $\left(z_{1}, \ldots, z_{p}\right) \in \mathbf{G}_{1} \Longleftrightarrow(\forall r>0):\left(r z_{1}, \ldots, r z_{p}\right) \in \mathbf{G}_{r}$;

iv) $\left(z_{1}, \ldots z_{p}\right) \in \mathbf{G}_{r} \Longrightarrow\left(\forall \theta=\left(\theta_{1}, \ldots, \theta_{p}\right) \in \mathbb{R}^{p}\right):\left(z_{1} e^{i \theta_{1}}, \ldots, z_{p} e^{i \theta_{p}}\right) \in \mathbf{G}_{r}$.

By $\mathbb{G}=\left\{\mathbf{G}=\left(\mathbf{G}_{r}\right)_{r \geq 0}: \mathrm{i}\right)$-iv $\left.)\right\}$ we denote the class the systems of such domains. Remark that following system $\mathbf{G}=\left(\mathbf{G}_{r}\right)_{r \geq 0}$ of the domains $\mathbf{G}_{r}$ is contained in the class $\mathbb{G}$ :

i) $\mathbf{G}_{r}=C_{r, a}=\left\{\left(z_{1}, \ldots, z_{p}\right) \in \mathbb{C}^{p}:\left|z_{j}\right|<a_{j} r, 1 \leq j \leq p\right\}$;

ii) $\mathbf{G}_{r}=\mathbb{B}_{r, a}=\left\{\left(z_{1}, \ldots, z_{p}\right) \in \mathbb{C}^{p}: a_{1}\left|z_{1}\right|^{2}+\ldots+a_{p}\left|z_{p}\right|^{2}<r^{2}\right\}$;

iii) $\mathbf{G}_{r}=\Pi_{r, a}=\left\{\left(z_{1}, \ldots, z_{p}\right) \in \mathbb{C}^{p}: a_{1}\left|z_{1}\right|+\ldots+a_{p}\left|z_{p}\right|<r\right\}$;

iv) $\mathbf{G}_{r}=\left\{\left(z_{1}, \ldots, z_{p}\right) \in \mathbb{C}^{p}:\left|z_{1}\right|^{a_{1}} \cdot \ldots \cdot\left|z_{2}\right|^{a_{p}}<r^{a_{1}+\cdots+a_{p}}\right\}$;

where $a=\left(a_{1}, \ldots, a_{p}\right), a_{j}>0(1 \leq j \leq p), r>0$.

For $r>0$ and an entire function $f \in \mathcal{E}^{1}(\lambda)$ we denote by $M_{f}(r)=\max \{|f(z)|:|z|=r\}$ the maximum modulus, and by $\mu_{f}(r)=\max \left\{\left|a_{k}\right| r^{\lambda_{k}}: k \geq 0\right\}$ the maximal term of power series (2). For $r>0$ and an entire function $f \in \mathcal{E}^{p}(\lambda)$ of the form (1) we denote

$$
M(r, f)=\max \left\{|f(z)|: z \in \overline{\mathbf{G}}_{r}\right\}, \quad m_{k}(r, f)=\max \left\{\left|P_{k}(z)\right|: z \in \overline{\mathbf{G}}_{r}\right\} \quad(k \geq 0) .
$$

By the maximum modulus principle there exists a point $z^{(k)}=\left(z_{1}^{(k)}, \ldots, z_{p}^{(k)}\right) \in \partial \mathbf{G}_{r}$ such that $m_{k}(r, f)=\left|P_{k}\left(z^{(k)}\right)\right|$. The definition of $\mathbf{G}_{r}$ implies $s^{(k)}=\left(s_{1}^{(k)}, \ldots, s_{p}^{(k)}\right) \stackrel{\text { def }}{=} \frac{z^{(k)}}{r} \in \partial \mathbf{G}_{1}$, but $P_{k}(z)$ is a homogeneous polynomial. Hence $P_{k}\left(z^{(k)}\right)=r^{\lambda_{k}} P_{k}\left(s^{(k)}\right)$. Thus $\left|P_{k}\left(s^{(k)}\right)\right|=$ $\max \left\{\left|P_{k}(z)\right|: z \in \overline{\mathbf{G}}_{1}\right\}=m_{k}(1, f)$ and therefore $s^{(k)}$ does not depend on $r$. So,

$$
m_{k}(r, f)=r^{\lambda_{k}}\left|P_{k}\left(s^{(k)}\right)\right| \quad(r>0, k \geq 0) .
$$

According to [1] define now the diagonal maximal term of the series (1)

$$
m(r, f) \stackrel{\text { def }}{=} \max \left\{m_{k}(r, f): k \geq 0\right\}=\max \left\{r^{\lambda_{k}} m_{k}(1, f): k \geq 0\right\} .
$$

We remark that $m(r, f) \equiv \mu_{f}(r)$ in the case $p=1$. 
Let $n(t)=\sum_{\lambda_{k} \leq t} 1$ be the counting function of the sequence $\lambda=\left(\lambda_{k}\right)$. From Theorem 1 in the paper [2], proved for entire Dirichlet series, it follows such a statement. If a sequence $\lambda=\left(\lambda_{k}\right)$ satisfies the condition

$$
\varlimsup_{t \rightarrow+\infty} \frac{\ln (n(t+\sqrt{\psi(t)})-n(t-\sqrt{\psi(t)}))}{\ln t} \leq p_{1}<+\infty
$$

for some positive function $\psi: \mathbb{R}_{+} \rightarrow \mathbb{R}_{+}$such that $\int_{0}^{+\infty} d t / \psi(t)<+\infty$, then for every entire function $f \in \mathcal{E}^{1}(\lambda)$ and any $\varepsilon>0$ there exists a set $E=E(\varepsilon, f) \subset[1,+\infty)$ of finite logarithmic measure (i.e., $\left.\ln -\operatorname{meas}(E) \stackrel{\text { def }}{=} \int_{E} d \ln r<+\infty\right)$ such that

$$
M_{f}(r) \leq C \mu_{f}(r)\left(\ln \mu_{f}(r)\right)^{p_{1}+\varepsilon}
$$

holds for all $r \in[1,+\infty) \backslash E$. Here $C$ is some constant depending only on $f$ and $\varepsilon$. Hence, in particular, it follows (see also [3, 4]), that if

$$
(\exists \triangle \in(0,+\infty))(\exists \varrho \in[1 / 2,1])(\exists D>0)\left(\exists t_{0}>0\right)\left(\forall t>t_{0}\right):\left|n(t)-\triangle t^{\varrho}\right| \leq D,
$$

then inequality (4) holds with $p_{1}=(2 \varrho-1) / 2$, because in this case condition $(3)$ is satisfied with $p_{1}=(2 \varrho-1) / 2$. In the case $f \in \mathcal{E}^{1}$, i.e. $\lambda_{k} \equiv k(k \geq 0)$, condition (5) holds with $\varrho=1$. Therefore, inequality (4) holds with $p_{1}=1 / 2$, i.e., we have the classical Wiman's inequality (see $[5,6,7])$.

In [2] it is also proved that for every sequence $\lambda=\left(\lambda_{k}\right)$ such that there exists a continuous positive increasing to $+\infty$ in the interval $[0,+\infty)$ function $\psi$ satisfying $\psi(t)=O\left(t \ln t \ln ^{2} \ln t\right)$ $(t \rightarrow+\infty), \int_{0}^{+\infty} \frac{d t}{\psi(t)}<+\infty$ and

$$
\left(\exists p_{1}>0\right): \quad \varliminf_{t \rightarrow+\infty} t^{-p_{1}}(n(t+\sqrt{\psi(t)})-n(t-\sqrt{\psi(t)}))>0,
$$

there exists an entire function $f \in \mathcal{E}^{1}(\lambda)$ such that

$$
\frac{M_{f}(r)}{\mu_{f}(r)}\left(\ln \mu_{f}(r)\right)^{-p_{1}} \rightarrow+\infty \quad(r \rightarrow+\infty)
$$

Condition (5) implies that $(6)$ holds with $p_{1}=(2 \varrho-1) / 2$. Thus, some entire function satisfies relation $(7)$ with $p_{1}=(2 \varrho-1) / 2$. It follows from the foregoing that if condition (5) is satisfied, then there exists a function $f \in \mathcal{E}^{1}(\lambda)$ such that relations (4) and (7) hold with $p_{1}=(2 \varrho-1) / 2$. In particular, for some entire function $f \in \mathcal{E}^{1}$ we have

$$
\frac{M_{f}(r)}{\mu_{f}(r) \sqrt{\ln \mu_{f}(r)}} \rightarrow+\infty \quad(r \rightarrow+\infty) .
$$

This means that we cannot replace $(2 \varrho-1) / 2$ in $(4)$ by a smaller number. Moreover, we cannot even replace $\varepsilon>0$ in (4) on $\varepsilon=0$. Hence we get in Wiman's inequality for the class of all entire functions $\mathcal{E}^{1}$ the number $\frac{1}{2}$ cannot be replaced by a smaller number. Moreover, $\varepsilon>0$ cannot be replaced by $\varepsilon=0$. We note also that $M_{f}(r) \sim \sqrt{2 \pi} \mu_{f}(r) \sqrt{\ln \mu_{f}(r)}(r \rightarrow+\infty)$ for the entire function $f(z)=e^{z}$. 
Theorem on Wiman's type inequality with diagonal maximal term of the series for the class $\mathcal{E}^{2}$ and exhausting $\mathbb{C}^{2}$ by an arbitrary system of complete multiple-circular domains is proved in the paper [1].

Theorem A ([1, p.33, Theorem 3]). For every entire function $f \in \mathcal{E}^{2}$ and for any $\varepsilon>0$ there exist a constant $C=C(\varepsilon, f)>0$ and a set $E \subset[1,+\infty]$ of finite logarithmic measure such that the inequality

$$
M(r, f) \leq C \cdot m(r, f)(\ln m(r, f))^{\frac{1}{2}+\varepsilon}
$$

holds for all $r \in[1,+\infty] \backslash E$.

In this paper we prove analogues of cited above results ([2]) for the class $\mathcal{E}^{p}(\lambda)$. Obtained results in particular contain the result of Theorem A and their sharpness is also proved.

Note, that in [1] it is proved an analogue of the Wiman inequality for maximum modulus on bi-circle $M(r, f)=\max \left\{\left|f\left(z_{1}, z_{2}\right)\right|:\left|z_{1}\right|=r_{1},\left|z_{2}\right|=r_{2}\right\}$ and maximal term $\mu(r, f)=$ $\max \left\{\left|a_{n}\right| r^{n}: n \in \mathbb{Z}_{+}^{2}\right\}, r=\left(r_{1}, r_{2}\right) \in \mathbb{R}_{+}^{2}$ with $3 / 2$ instead of $1 / 2$ in (8). A. Schumitski ([8, 9]), P. Fenton ([10]), O. Skaskiv and O. Trakalo ([11]) and some others authors have improved Bitlyan and Goldberg's result as in the specification of inequality, and in the specification of describing exceptional set, and also established analogues Wiman's inequality for other classes analytic functions of several variables (see also [12]-[26]).

\section{Main results.}

2.1. Wiman's type inequality for entire gap power series and diagonal maximal term. Let $\mathcal{L}$ be the class of positive continuous functions $\psi: \mathbb{R}_{+} \rightarrow \mathbb{R}_{+}$such that $\int_{0}^{+\infty} \frac{d x}{\psi(x)}<$ $+\infty$.

First we prove such assertions.

Theorem 1. If a sequence $\lambda=\left(\lambda_{k}\right)$ satisfies the condition

$$
\left(\exists p_{1} \in(0,+\infty)\right)\left(\exists t_{0}>0\right)\left(\forall t \geq t_{0}\right): \quad n(t+\sqrt{\psi(t)})-n(t-\sqrt{\psi(t)}) \leq t^{p_{1}}
$$

for some function $\psi \in \mathcal{L}$, then for every entire function $f \in \mathcal{E}^{p}(\lambda), p \geq 2$ and for any $\varepsilon>0$ there exist a constant $C=C(\varepsilon, f)>0$ and a set $E=E(\varepsilon, f) \subset[1,+\infty)$ of finite logarithmic measure such that the inequality

$$
M(r, f) \leq C m(r, f)(\ln m(r, f))^{p_{1}}(\ln \ln m(r, f))^{p_{1}+\varepsilon}
$$

holds for all $r \in[1,+\infty] \backslash E$.

Theorem 2. Let $\psi \in \mathcal{L}$ be an increasing in interval $[0,+\infty)$ function such that $\psi(t)=$ $O\left(t \ln t \ln ^{2} \ln t\right)(t \rightarrow+\infty)$, and for a sequence $\lambda=\left(\lambda_{k}\right)$ the condition

$$
\left(\exists p_{1}>0\right)\left(\exists C_{1}>0\right)\left(\exists t_{0}>0\right)\left(\forall t \geq t_{0}\right): n(t+\sqrt{\psi(t)})-n(t-\sqrt{\psi(t)}] \geq C_{1} t^{p_{1}}
$$

holds. Then for every $\varepsilon \in\left(0, p_{1}\right)$ there exists an entire function $f \in \mathcal{E}^{p}(\lambda)$ such that

$$
\frac{M(r, f)}{m(r, f) \ln ^{p_{1}} m(r, f) \ln ^{p_{1}-\varepsilon} \ln m(r, f)} \rightarrow+\infty \quad(r \rightarrow+\infty)
$$

Remark 1. In the case of assumption (5) instead of (8) and (9) the assertions of Theorems 1 and 2 were announced in [28]. 
Proof of Theorem 1. Let $z=r s, z_{1}=r s_{1}, \ldots, z_{p}=r s_{p}, r>0,\left(P_{k}\right)$ be a homogenous polynomial, so we have

$$
f(z)=\sum_{k=0}^{+\infty} r^{\lambda_{k}} P_{k}(s) .
$$

Hence, using $m_{k}(1, f)=\left|P_{k}\left(s^{(k)}\right)\right|=\max \left\{\left|P_{k}(z)\right|: z \in \bar{G}_{1}\right\}$, we obtain

$$
\begin{aligned}
& M(r, f)=\max \left\{|f(z)|: z \in \bar{G}_{r}\right\}=\max \left\{|f(r s)|: s \in \bar{G}_{1}\right\} \leq \\
& \leq \sum_{k=0}^{+\infty} r^{\lambda_{k}} \max \left\{\left|P_{k}(s)\right|: s \in \bar{G}_{1}\right\}=\sum_{k=0}^{+\infty} r^{\lambda_{k}} m_{k}(1, f) \stackrel{\text { def }}{=} H(r) .
\end{aligned}
$$

Here $H(r)$ is an entire function of one variable, for which condition (8) of Theorem 1 holds.

Now we need one result from [7, Theorem 1].

Let $\mathcal{I}(\nu)$ be the class of the functions $F: \mathbb{R} \rightarrow \mathbb{R}_{+}$defined by the integral of the form

$$
F(x)=\int_{\mathbb{R}_{+}} a(u) e^{x u} \nu(d u)
$$

where $\nu$ is a countable additive measure on the $\sigma$-algebra $\mathcal{B}\left(\mathbb{R}_{+}\right)$of Borel sets on $\mathbb{R}_{+}$(Borel measure) such that $\nu(\{x: 0 \leq x \leq b\})<+\infty$ for any $b>0, a: \mathbb{R}_{+} \rightarrow \mathbb{R}_{+}$be a positive measurable function. Denote by $\operatorname{supp} \nu$ the support of the measure $\nu$, i.e. the closed set $E=: \operatorname{supp} \nu$ such that $\nu(\mathbb{R} \backslash E)=0$ and $\nu\left(\left\{u \in \mathbb{R}:\left|u-u_{0}\right|<r\right\}\right)>0$ for any $u_{0} \in E$ and $r>0$. For $x \in \mathbb{R}$ and $F \in \mathcal{I}(\nu)$ we set

$$
\mu_{*}(x)=\sup \left\{a(u) e^{x u}: u \in \operatorname{supp} \nu\right\}, \quad \mu^{*}(x)=\sup \left\{a(u) e^{x u}: u \in \mathbb{R}\right\} .
$$

Lemma 1 ([7], Theorem 1). Let $F \in \mathcal{I}(\nu)$. If

$$
(\exists \psi \in \mathcal{L})\left(\exists p_{1}<+\infty\right)\left(\exists t_{0}\right)\left(\forall t \geq t_{0}\right): \quad \nu(t-\sqrt{\psi(t)}, t+\sqrt{\psi(t)}] \leq t^{p_{1}},
$$

then for every $\varepsilon>0$ there exists a set $E_{1} \subset \mathbb{R}_{+}$of finite Lebesgue measure, i.e. meas $\left(E_{1}\right):=$ $\int_{E_{1}} d x<+\infty$, such that for all $x \in[0,+\infty) \backslash E$

$$
F(x) \leq C \mu_{*}(x) \ln ^{p_{1}} \mu_{*}(x) \ln _{2}^{p_{1}+\varepsilon} \mu_{*}(x) .
$$

Here $C$ is some constant depending only on $F$ and $\varepsilon, \ln _{2} t:=\ln (\ln t)$.

Let

$$
\nu(E)=\sum_{k=0}^{+\infty} \delta_{\lambda_{k}}(E),
$$

where $\delta_{\lambda}(E)=1$ for $\lambda \in E, \delta_{\lambda}(E)=0$ for $\lambda \notin E$ for every bounded set $E \subset \mathbb{R}_{+}$. We put $a(u)=m_{k}(1, f)$ for $u=\lambda_{k}, a(u)=0$ for $u \in \mathbb{R}_{+} \backslash\left\{\lambda_{k}\right\}$. Then

$$
H\left(e^{x}\right)=\int_{\mathbb{R}_{+}} a(u) e^{u x} \nu(d u), \quad \mu_{h}\left(e^{x}\right)=\mu_{*}(x) \quad(x>0) .
$$

Now from Lemma 1 it follows

$$
\begin{gathered}
M\left(e^{x}, f\right) \leq H\left(e^{x}\right) \leq C \mu_{*}(x) \ln ^{p_{1}} \mu_{*}(x) \ln _{2}^{p_{1}+\varepsilon / 2} \mu_{*}(x)= \\
=C \mu_{H}\left(e^{x}\right) \ln ^{p_{1}} \mu_{H}\left(e^{x}\right) \ln _{2}^{p_{1}+\varepsilon / 2} \mu_{H}\left(e^{x}\right)=C m\left(e^{x}, f\right) \ln ^{p_{1}} m\left(e^{x}, f\right) \ln _{2}^{p_{1}+\varepsilon / 2} m\left(e^{x}, f\right)
\end{gathered}
$$


for all $x \in \mathbb{R}_{+} \backslash E_{1}$, meas $\left(E_{1}\right)<+\infty$. We put $r=e^{x}$ and denote $E=e^{E_{1}}$. Then we obtain that inequality (1) holds for all $r \in[1,+\infty) \backslash E$,

$$
\ln -\operatorname{meas}(E)=\int_{E} d \ln r=\int_{E_{1}} d x=\operatorname{meas}\left(E_{1}\right)<+\infty .
$$

Proof of Theorem 2. Remark that for an arbitrary entire $f \in \mathcal{E}^{p}$ of the form

$$
f(z)=\sum_{k=0}^{+\infty} a_{k}\left(z_{1} \cdot \ldots \cdot z_{p}\right)^{\lambda_{k}}, \quad z=\left(z_{1}, \ldots, z_{p}\right) \in \mathbb{C}^{p}
$$

we have

$$
m_{k}(r, f)=\max \left\{\left|a_{k}\right|\left|z_{1} \cdot \ldots \cdot z_{p}\right|^{\lambda_{k}}:\left(z_{1}, \ldots, z_{p}\right) \in \bar{G}_{r}\right\} .
$$

Denote $d_{k}=\max \left\{\left|s_{1} \cdot \ldots \cdot s_{p}\right|^{\lambda_{k}}:\left(s_{1}, \ldots, s_{p}\right) \in \bar{G}_{1}\right\}(k \geq 1)$. Then

$$
m_{k}(r, f)=d_{k} a_{k} r^{p \lambda_{k}}, \quad(k \geq 1),
$$

and

$$
m(r, f)=\max \left\{m_{k}(r, f): k \geq 1\right\}=\max \left\{d_{k} a_{k} r^{p \lambda_{k}}: k \geq 1\right\} .
$$

We make use of the following assertion.

Lemma 2 ([7], Theorem 2). Let $\psi \in \mathcal{L}$ be such that $\psi(t)=O\left(t \ln t \ln _{2}^{2} t\right)(t \rightarrow+\infty)$ and a measure $\nu$ such that $\ln \nu(0, t]=O(t)(t \rightarrow+\infty)$ and

$$
(\exists C>0)\left(\exists p_{1}>0\right)\left(\exists t_{0}>0\right)\left(\forall t \geq t_{0}\right): \nu(t-\sqrt{\psi(t)}, t+\sqrt{\psi(t)}] \geq C t^{p_{1}} .
$$

Then for every $\varepsilon \in\left(0, p_{1}\right)$ there exists $F \in \mathcal{I}(\nu)$ of form (10) such that

$$
\frac{F(x)}{\mu^{*}(x) \ln ^{p_{1}} \mu^{*}(x) \ln _{2}^{p_{1}-\varepsilon} \mu^{*}(x)} \rightarrow+\infty,(x \rightarrow+\infty) .
$$

Let again

$$
\nu(E)=\sum_{k=0}^{+\infty} \delta_{\lambda_{k}^{(1)}}(E), \quad \lambda_{k}^{(1)}:=p \lambda_{k} .
$$

Condition (9) for a sequence $\left(\lambda_{k}\right)$ holds with a function $\psi \in \mathcal{L}$ if and only if it is holds for $\left(b \lambda_{k}\right), b>0$, with the function $\psi_{1}(u)=b^{2} \psi(u / b)$ instead of the function $\psi$, and the constant $C b^{-p_{1}}$ instead of constant $C$. Therefore, condition (14) holds for such $\nu$, so Lemma 2 implies that there exists $F \in \mathcal{I}(\nu)$ of form (10) such that relation (15) is valid.

Now we put $h_{k}:=a\left(\lambda_{k}^{(1)}\right)=a\left(p \lambda_{k}\right)$. Then

$$
H(r):=\sum_{k=1}^{+\infty} h_{k} r^{p \lambda_{k}}=\int_{1}^{+\infty} a(u) e^{u \ln r} \nu(d u)=F(\ln r), \quad \mu^{*}(\ln r) \geq \mu_{H}(r) \quad(r \geq 1) .
$$

Thus $h: \mathbb{C} \rightarrow \mathbb{C}$ is an entire function of one variable. From (15) follows

$$
\frac{H(r)}{\mu^{*}(\ln r) \ln ^{p_{1}} \mu^{*}(\ln r) \ln _{2}^{p_{1}-\varepsilon} \mu^{*}(\ln r)} \rightarrow+\infty \quad(r \rightarrow+\infty) .
$$


We set $a_{k}=h_{k} / d_{k}(k \geq 0)$ and consider a function $f$ of the form (11). Remark, that for $r>0$ and each $z \in \partial \bar{G}_{1}$

$$
M(r, f) \geq|f(r z)|
$$

We put $z=(1, \ldots, 1) \in \mathbb{R}_{+}^{p}$. Without loss of generality, we may suppose that $z=(1, \ldots, 1) \in$ $\partial \bar{G}_{1}$. Then, for all $r>0$ we get

$$
M(r, f) \geq|f(r z)|=\sum_{k=0}^{+\infty} h_{k} r^{p \lambda_{k}}=H(r) .
$$

From equalities (12), (13) it follows that

$$
m(r, f)=\max \left\{a_{k} d_{k} r^{p \lambda_{k}}: k \geq 0\right\}=\max \left\{h_{k} r^{p \lambda_{k}}: k \geq 0\right\}=\mu_{H}(r) \leq \mu^{*}(\ln r) \quad(r \geq 1) .
$$

Therefore, relation (16) implies

$$
\frac{M(r, f)}{m(r, f) \ln ^{p_{1}} m(r, f) \ln _{2}^{p_{1}-\varepsilon} m(r, f)} \geq \frac{H(r)}{\mu^{*}(\ln r) \ln ^{p_{1}} \mu^{*}(\ln r) \ln _{2}^{p_{1}-\varepsilon} \mu^{*}(\ln r)} \rightarrow+\infty
$$

as $r \rightarrow+\infty$. We suppose now that $z=(1, \ldots, 1) \notin \partial \bar{G}_{1}$, and $r_{1}>0$ such that $z=$ $\left(r_{1}, \ldots, r_{1}\right) \in \partial \bar{G}_{1}$. Consider the function $f_{1}(z)=f\left(z r_{1}\right)$ and the exhaustion by the domains $\bar{G}_{r}^{0}:=\bar{G}_{r r_{1}}$. Then, $z=(1, \ldots, 1) \in \partial \bar{G}_{1}^{0}$,

$$
M\left(r, f_{1}, G^{0}\right)=M(r, f, G), \quad m\left(r, f_{1}, G^{0}\right)=m(r, f, G) .
$$

Since $M(r, f)=M(r, f, G)$ and $M\left(r, f_{1}\right)=M\left(r, f_{1}, G^{0}\right)$ for $r>0$,

$$
\frac{M(r, f)}{m(r, f) \ln ^{p_{1}} m(r, f) \ln _{2}^{p_{1}-\varepsilon} m(r, f)}=\frac{M\left(r, f_{1}\right)}{m\left(r, f_{1}\right) \ln ^{p_{1}} m\left(r, f_{1}\right) \ln _{2}^{p_{1}-\varepsilon} m\left(r, f_{1}\right)} \rightarrow+\infty
$$

as $r \rightarrow+\infty$, because for the function $f_{1}$ and the domains $G_{r}^{0}$ relation (17) holds.

2.2. Bitlyan-Goldberg type inequality and new description of exceptional set. We will adhere to the general scheme of reasoning from the previous subsection 2.1. First we get one statement containing a new estimate of the exceptional set of functions from class $\mathcal{I}(\nu)$.

Theorem 3. Let $F \in \mathcal{I}(\nu)$ and $\nu$ be a Borel measure such that $\left(\exists t_{0} \geq 0\right)\left(\exists c_{2}, c_{3}>0\right)(\forall T \geq$ $\left.t_{0}\right)\left(\forall t \in\left[t_{0}, T\right]\right):$

$$
\nu(T-t, T+t] \leq c_{2} t+c_{3} .
$$

If $h$ is a positive function such that $\int_{0}^{+\infty} h(x) d x=+\infty$ and $\ln _{2}^{+} h(x)=o\left(\ln _{2} F(x)\right)(x \rightarrow$ $+\infty)$, then for each $\varepsilon>0$ there exists a set $E_{3}(\varepsilon, F, h) \equiv E_{3}$ such that $h$-meas $E_{3}:=$ $\int_{E_{3}} h(x) d x<+\infty$ and the inequality

$$
F(x) \leq h(x) \mu_{*}(x)\left(\ln \mu_{*}(x)\right)^{1 / 2+\varepsilon}
$$

holds for every $x \in[0 ;+\infty) \backslash E_{3}$.

We need the following assertion. 
Lemma 3 ([29]). Let $g_{1}(x)$ be a positive differentiable non-decreasing on $[0 ;+\infty)$ function, $\psi(x)$ be a positive continuous increasing on $[0 ;+\infty)$ function such that $\int_{0}^{+\infty} \frac{d x}{\psi(x)}<+\infty$, and $h_{0}(x)$ be a positive local integrable on $[0 ;+\infty)$ function such that $\int_{0}^{+\infty} h_{0}(x) d x=+\infty$. Then there exists a set $E_{0} \subset[0 ;+\infty)$ such that $h$-meas $E_{0}:=\int_{E_{0}} h_{0}(x) d x<+\infty$ and for every $x \in[0 ;+\infty) \backslash E_{0}$

$$
g_{1}^{\prime}(x) \leq h_{0}(x) \psi\left(g_{1}(x)\right) .
$$

Proof of Theorem 3. We put $g(x)=\ln F(x)$. As in [29] for fixed $x \in \mathbb{R}$ we have

$$
F(x) \leq 2 \int_{\left|u-g^{\prime}(x)\right|<\sqrt{2 g^{\prime \prime}(x)}} f(u) e^{x u} \nu(d u) .
$$

Thus by the hypotheses of Theorem 3

$$
F(x) \leq 2 \mu_{*}(x) \nu\left(g^{\prime}(x)-\sqrt{2 g^{\prime \prime}(x)}, g^{\prime}(x)+\sqrt{2 g^{\prime \prime}(x)}\right] \leq 2 \mu_{*}(x)\left(c_{2} \sqrt{2 g^{\prime \prime}(x)}+c_{3}\right) .
$$

Let for $\varepsilon_{1}>0$ and $\varepsilon_{2}>0$

$$
\begin{gathered}
E_{1}=\left\{x>0: g^{\prime \prime}(x)>h(x) g^{\prime}(x)\left(\ln g^{\prime}(x)\right)^{1+\varepsilon_{1}}, g^{\prime}(x) \geq 2,\right. \\
E_{2}=\left\{x>0: g^{\prime}(x)>h(x)(g(x))^{1+\varepsilon_{2}}, g(x) \geq 1\right\} .
\end{gathered}
$$

Now we use the statement of the Lemma 3 twice. First, choosing $g_{1}(x)=g^{\prime}(x), \psi(x)=$ $2 c_{2}^{2} x(\ln x)^{1+\varepsilon_{1}} /\left(2 c_{2}^{2}\right)$, and then $g_{1}(x)=g(x), \psi(x)=x^{1+\varepsilon_{2}}$, we obtain that

$$
h \text {-meas } E_{1}<+\infty, h \text {-meas } E_{2}<+\infty \text {, }
$$

i.e. $h$-meas $E_{1} \cup E_{2}<+\infty$.

Therefore for $x \notin E:=E_{1} \cup E_{2}$ we have

$$
F(x) \leq 2 \mu_{*}(x)\left(h(x)(g(x))^{\left(1+\varepsilon_{2}\right) / 2}\left(\ln \left(h(x)(g(x))^{1+\varepsilon_{2}}\right)\right)^{\left(1+\varepsilon_{1}\right) / 2}+c_{3}\right) .
$$

By the assumptions of Theorem $3 \ln h(x)<(\ln F(x))^{\delta}\left(x \geq x_{0}(\delta)\right)$ for every $\delta>0$. Therefore,

$$
\begin{gathered}
\left(\ln \left(h(x)(g(x))^{1+\varepsilon_{2}}\right)\right)^{\left(1+\varepsilon_{1}\right) / 2} \leq \\
\leq\left((\ln F(x))^{\delta}+\left(1+\varepsilon_{2}\right) \ln _{2} F(x)\right)^{\left(1+\varepsilon_{1}\right) / 2} \leq(1+o(1))(\ln F(x))^{\delta\left(1+\varepsilon_{1}\right) / 2}
\end{gathered}
$$

as $x \rightarrow+\infty$. Hence

$$
F(x) \leq 2 \mu_{*}(x)\left(h(x)(\ln F(x))^{(1+\varepsilon) / 2}+c_{3}\right) \quad(x \rightarrow+\infty, x \notin E),
$$

where $\varepsilon=\varepsilon_{2}+\delta\left(1+\varepsilon_{1}\right)$.

We note that $h(x) \leq \exp \left\{\ln ^{1 / 3} F(x)\right\}(x \rightarrow+\infty)$. Thus

$$
h(x)(\ln F(x))^{(1+\varepsilon) / 2}+c_{3} \leq \exp \left\{\ln ^{2 / 3} F(x)\right\} \quad(x \rightarrow+\infty)
$$

and as $x \rightarrow+\infty(x \notin E)$

$$
\begin{gathered}
\ln F(x) \leq \ln 2+\ln \mu_{*}(x)+\ln \left(h(x)(\ln F(x))^{(1+\varepsilon) / 2}+c_{3}\right) \leq \\
\leq \ln 2+\ln \mu_{*}(x)+\ln ^{2 / 3} F(x) .
\end{gathered}
$$


hence $\ln F(x) \leq(1+o(1)) \ln \mu_{*}(x)$. Consequently,

$$
F(x) \leq 2 \mu_{*}(x)\left(h(x)(\ln F(x))^{(1+\varepsilon) / 2}+c_{3}\right) \leq 4 \mu_{*}(x) h(x)\left(\ln \mu_{*}(x)\right)^{(1+\varepsilon) / 2}
$$

as $x \rightarrow+\infty(x \notin E)$.

We now consider the general case of entire functions from the class $\mathcal{E}^{p}:=\mathcal{E}^{p}(\lambda)$ with $\lambda_{k} \equiv k \in \mathbb{Z}_{+}$.

Theorem 3 implies the following corollary.

Theorem 4. Let $f \in \mathcal{E}^{p}$. If a positive local integrable on $[1 ;+\infty)$ function $h_{0}$ is such that $\int_{1}^{+\infty} h_{0}(r) d \ln r=+\infty$ and $\ln ^{+} \ln h_{0}(r)=o(\ln \ln m(r, f))(r \rightarrow+\infty)$, then for each $\varepsilon>0$ there exists a set $E_{4}(\varepsilon, f, h) \equiv E_{4}$ such that $h_{0}$-log-meas $E_{4}:=\int_{E_{4}} h_{0}(r) d \ln r<+\infty$ and

$$
M(r, f) \leq h_{0}(r) m(r, f)(\ln m(r, f))^{1 / 2+\varepsilon}
$$

holds for all $r \in[1,+\infty) \backslash E_{4}$.

Proof. First, we reason as in the proof of Theorem 1 up to the application of Lemma 1. Next, applying instead of Lemma 1 the statement of Lemma 3, we obtain the required inequality and the required description of the exceptional set, because

$$
\int_{E_{4}} h_{0}(r) d \ln r=\int_{E_{3}} h_{0}\left(e^{x}\right) d x=\int_{E_{3}} h(x) d x<+\infty,
$$

where the set $E_{4}$ is the image of the set $E_{3}$ by mapping $r=e^{x}: E_{3} \rightarrow E_{4}$.

Note now, that for the sequence $\lambda_{k} \equiv k$ one has $n(T+t)-n(T-t) \leq 2 t+1$, i.e. conditions (18) take place with $c_{2}=2, c_{3}=1$. It remains to obtain from the condition $\ln ^{+} \ln h_{0}(r)=o(\ln \ln m(r, f))(r \rightarrow+\infty)$ condition $\ln _{2}^{+} h(x)=o\left(\ln _{2} F(x)\right)(x \rightarrow+\infty)$ with $h(x)=h_{0}\left(e^{x}\right), F(x)=H\left(e^{x}\right)$. But, by Cauchy's inequality $m(r, f)=\mu_{H}(r) \leq H(r)$.

If in Theorem 4 we choose $h_{0}(r)=\ln ^{\varepsilon} m(r, f)$, then we immediately obtain the following statement.

Corollary. If $f \in \mathcal{E}^{p}$, then for each $\varepsilon>0$ there exists a set $E_{5}(\varepsilon, f, h) \equiv E_{5}$ such that $\int_{E_{5}} \ln ^{\varepsilon} m(r, f) d \ln r<+\infty$ and the inequality

$$
M(r, f) \leq m(r, f)(\ln m(r, f))^{1 / 2+\varepsilon}
$$

holds for all $r \in[1,+\infty) \backslash E_{5}$.

Remark, that in the case of entire functions of one complex variables statements similarly to Theorem 4 and Corollary 1 we find in [30] (see also [31]).

\section{REFERENCES}

1. Bitlyan I.F., Goldberg A.A. Wiman-Valiron's theorem for entire functions of several complex variables// Vestn. Leningrad univ. ser. mat., mech. and astr. - 1959. - V.2, №13. - P. 27-41. (in Russian) 
2. O.B. Skaskiv, On the classical Wiman's inequality for entire Dirichlet series, Visn. Lviv. Univer., ser. mekh.-mat. - 1999. - V.54. - P. 180-182. (in Ukrainian)

3. M.M. Sheremeta, Wiman-Valiron's method for entire functions, represented by Dirichlet series, Dokl. USSR Acad. Sci. - 1978. - V.240, №5. - P. 1036-1039. (in Russian)

4. Skaskiv O.B. Random gap power series and Wiman's inequality// Mat. Stud. - 2008. - V.30, №1. P. 101-106. (in Ukrainian)

5. Valiron G. Functions analytiques. - Paris: Press Univer. de France, 1954.

6. Wittich H. Neuer Untersuchungen über eindeutige analytische Funktionen. - Berlin-GöttingenHeidelberg: Springer-Verlag, 1955.

7. Kuryliak A.O., Ovchar I.E., Skaskiv O.B. Wiman's inequality for Laplace integrals// Int. Journal of Math. Analysis. - 2014. - V.8, №8. - P. 381-385.

8. Schumitsky A. Wiman-Valyron theory for entire functions of several complex variables. - Ph.D. Thesis, Cornell Univ., 1965.

9. Shumitzky A. A probabilistic approach to the Wiman-Valiron's theory for entire functions of several complex variables// Complex Variables. - 1989. - V.13. - P. 85-98.

10. Fenton P.C. Wiman-Valiron theory in two variables// Trans. Amer. Math. Soc. - 1995.- V.347, №11. P. 4403-4412.

11. Skaskiv O.B., Trakalo O.M. On classical Wiman's inequality for entire Dirichlet series// Mat. metods and fys.-mekh. polya. - 2000. - V.43, №3. - P. 34-39. (in Ukrainian)

12. Zrum O.V., Skaskiv O.B. On Wiman's inequality for random entire functions of two variables// Mat. Stud. - 2005. - V.23, №2. - P. 149-160. (in Ukrainian)

13. Skaskiv O.B., Zrum O.V. Wiman-type inequalities for entire functions of two complex variables with rapidly oscillating coefficients// Mat. Metody Phys.-Mekh. Polya. - 2005. - V.48, №4. - P. 78-87. (in Ukrainian)

14. Skaskiv O.B., Zrum O.V. Refinement of Fenton's inequality for entire functions of two complex variables// Mat. Visn. Nauk. Tov. Im. Shevchenka. - 2006. - V.3. - P. 56-68. (in Ukrainian)

15. Gopala Krishna J. Generalised inverse and probability techniques and some fundamental growth theorems in $\mathbb{C}^{k} / /$ J. Indian Math. Soc. - 1977. - V.41. - P. 203-219.

16. Gopala Krishna J., Nagaraja Rao I.H. Generalised inverse and probability techniques and some fundamental growth theorems in $\mathbb{C}^{k} / /$ Jour. of the Indian Math. Soc. - 1977. - V.41. - P. 203-219.

17. O.B. Skaskiv, Random gap power series and Wiman's inequality// Mat. Stud. - 2008. - V.30, №1. P. 101-106. (in Ukrainian)

18. Kuryliak A.O., Skaskiv O.B. Wiman's type inequalities without exceptional sets for random entire functions of several variables// Mat. Stud. - 2012. - V.38, №1. - P. 35-50.

19. Skaskiv O. B., Kuryliak A. O. Maximum modulus of entire functions of two variables and arguments of coefficients of double power series// Mat. Stud. - 2011. - V.36, №2. - P. 162-175.

20. Zrum O.V., Kuryliak A.O., Skaskiv O.B. Levy's phenomenon for entire functions of several variables// arXiv: 1307.6164v1 [math.CV] 23 Jul 2013. - 14 p.

21. Kuryliak A.O., Skaskiv O.B., Zrum O.V. Levy's phenomenon for entire functions of several variables// Ufa Math. J. - 2014. - T.6, №2. - P. 111-120.

22. Kuryliak A.O., Shapovalovska L.O., Skaskiv O.B. Wiman's type inequality for some double power series// Mat. Stud. - 2013. - V.39, №2. - P. 134-141.

23. Kuryliak A.O., Shapovalovska L.O. Wiman's type inequality for entire functions of several complex variables with rapidly oscillating coefficients// Mat. Stud. - 2015. - V.43, №1. - P. 16-26. doi: $10.15330 / \mathrm{ms} .43 .1 .16-26$

24. Kuryliak A.O., Skaskiv O.B., Shapovalovska L.O. Wiman's inequality for analityc functions in a bydisc// Visn. Lviv. Univ. - 2014. - Vyp.79. -P. 89-96 (2014) (in Ukrainian)

25. Kuryliak A.O., Shapovalovska L.O., Skaskiv O.B. Wiman's type inequality for analytic functions in a polydisc// Ukr. Mat. Zh. - 2016. -V.68, №.1. -P. 78-86 (2016) (in Ukrainian)

26. Kuryliak A.O., Tsvigun V.L. Wiman's type inequality for multiple power series in an unbounded cylinder domain// Mat. Stud. - 2018. - V.49, №1. - P. 29-51.

27. Kuryliak A., Skaskiv O., Skaskiv S. Levy's phenomenon for analytic functions on a polydisk// European Journal of Mathematics. - 2020. - V.6, №1. - P. 138-152 doi.org/10.1007/s40879-019-00363-2

28. Panchuk S.I., Skaskiv O.B. Lacunary multiple power series and Wiman's inequality// International conference dedicated to the 120th anniversary of Stefan Banach (Lviv, 17.09-21.09.2012): Abstract of Reports. - Lviv, 2012. - P. 172-173. 
29. Skaskiv O.B. On certain relations between the maximum modulus and the maximal term of an entire Dirichlet series, Math. Notes. - 1999. - V.66, №2. - P. 223-232. Transl. from Mat. Zametky. - 1999. V.66, №2. - P. 282-292.

30. Skaskiv O.B., Zrum O.V., On an exeptional set in the Wiman inequalities for entire functions// Mat. Stud. - 2004. - V.21, №1. - P. 13-24. (in Ukrainian)

31. Skaskiv O.B., Filevych P.V., On the size of an exceptional set in the Wiman theorem, Mat. Stud. - 1999. - V.12, №1. - P. 31-36. (in Ukrainian)

Ivan Franko National University of Lviv

Lviv, Ukraine

andriykuryliak@gmail.com

olskask@gmail.com

s.panchuk@lnu.edu.ua 